\title{
Abnormal systemic venous connection possibly associated with a persistent right umbilical vein; a case report Britt Nakstad $^{* \dagger 1}$ and Bjarne Smevik ${ }^{\dagger 2}$
}

\author{
Address: ${ }^{1}$ Department of Paediatrics, Rikshospitalet University Hospital, Oslo, Norway and ${ }^{2}$ Department of Paediatric Radiology, Rikshospitalet \\ University Hospital, Oslo, Norway \\ Email: Britt Nakstad* - britt.nakstad@ahus.no; Bjarne Smevik - bjarne.smevik@rikshospitalet.no \\ * Corresponding author †Equal contributors
}

Published: 29 April 2004

BMC Pediatrics 2004, 4:7

This article is available from: http://www.biomedcentral.com/I47I-243I/4/7

(C) 2004 Nakstad and Smevik; licensee BioMed Central Ltd. This is an Open Access article: verbatim copying and redistribution of this article are permitted in all media for any purpose, provided this notice is preserved along with the article's original URL.
Received: 23 December 2003

Accepted: 29 April 2004

\begin{abstract}
Background: Abnormal venous connections involving a persistent right umbilical vein are rare. In a minority of cases the liver is entirely bypassed and the condition is associated with multiple congenital malformations.

Case presentation: The described case illustrates a systemic venous drainage that was severely abnormal in a newborn girl with a truncus arteriosus type II congenital heart defect. Injection of contrast medium through the umbilical vein catheter revealed a very peculiar venous connection that passed anterio-laterally through the right hemithorax before crossing in an oblique fashion towards the superior vena cava.
\end{abstract}

Conclusions: This venous drainage may be the result of a persistent right umbilical vein connecting with the superior vena cava.

\section{Background}

An abnormal venous connection involving a persistent right umbilical vein is a rare entity found in $0,2 \%$ to $0,4 \%$ of foetuses undergoing screening prenatal ultrasonography $[1,2]$. The intrahepatic variant is observed most frequently $[3,4]$, and the foetuses are often normal. In a limited number of cases the liver is entirely bypassed. The majority of newborns with this variety have multiple congenital malformations $[3,5]$ and the unrestricted umbilical venous blood flow to the heart may result in congestive heart failure. An accurate description is important when an operation is needed for palliation or correction. We report a case of congenital heart defect with an abnormal venous system that may have been formed by a persistent right umbilical vein connecting to a right cardinal vein merging with the superior vena cava.

\section{Case presentation}

Intrauterine ultrasound examination revealed two vessels in the umbilical cord and an abnormal venous return to the heart as well as congenital heart disease. The patient was a $2885 \mathrm{~g}$ female newborn of 38 weeks' gestational age delivered vaginally with an Apgar score of 8/9. Her parents of ethnical Pakistani origin were first cousins. A slightly dysmorphic appearance was noted such as low-placed ears, a broad base of the nose and a nuchal fold. A capillary haemangioma was observed stretching along the entire spine from the sacral region to her forehead. Ultrasonographic examination of the cerebrum was normal. Radiographs revealed a lumbar hemivertebra. The thymus was present. Her chromosomes were normal $46 \mathrm{XX}$ with no 22q11 deletion. A distinct rough systolic murmur indicating structural heart disease was heard and echocardiography of the heart confirmed a truncus arteriosus type II with a quadricuspid valve, an atrial septal defect as well as 


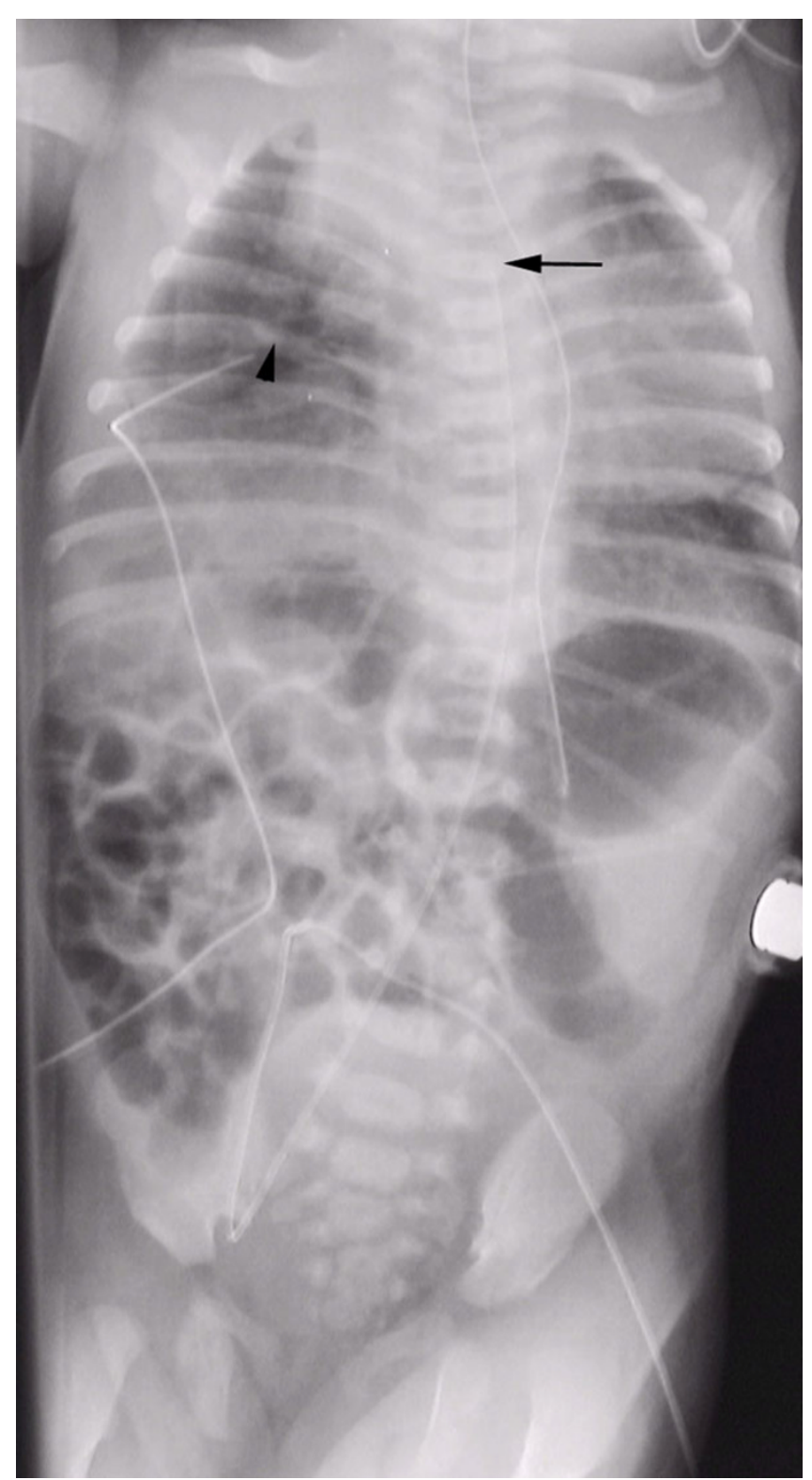

\section{Figure I}

Catheter in the umbilical artery courses in a normal fashion via the right iliac artery up into the descending aorta with the tip at the level of Th3 (arrow). Catheter inserted in the umbilical vein goes towards the right costo-phrenic angle and turns with the tip in a medial direction (arrowhead)

a ventricular septal defect and the pulmonary arteries originating from the ascending aorta. A very small right-sided jugular vein was demonstrated as well as a great venous structure posterior and lateral to the liver, interpreted as an azygos vein with the absence of the inferior vena cava.

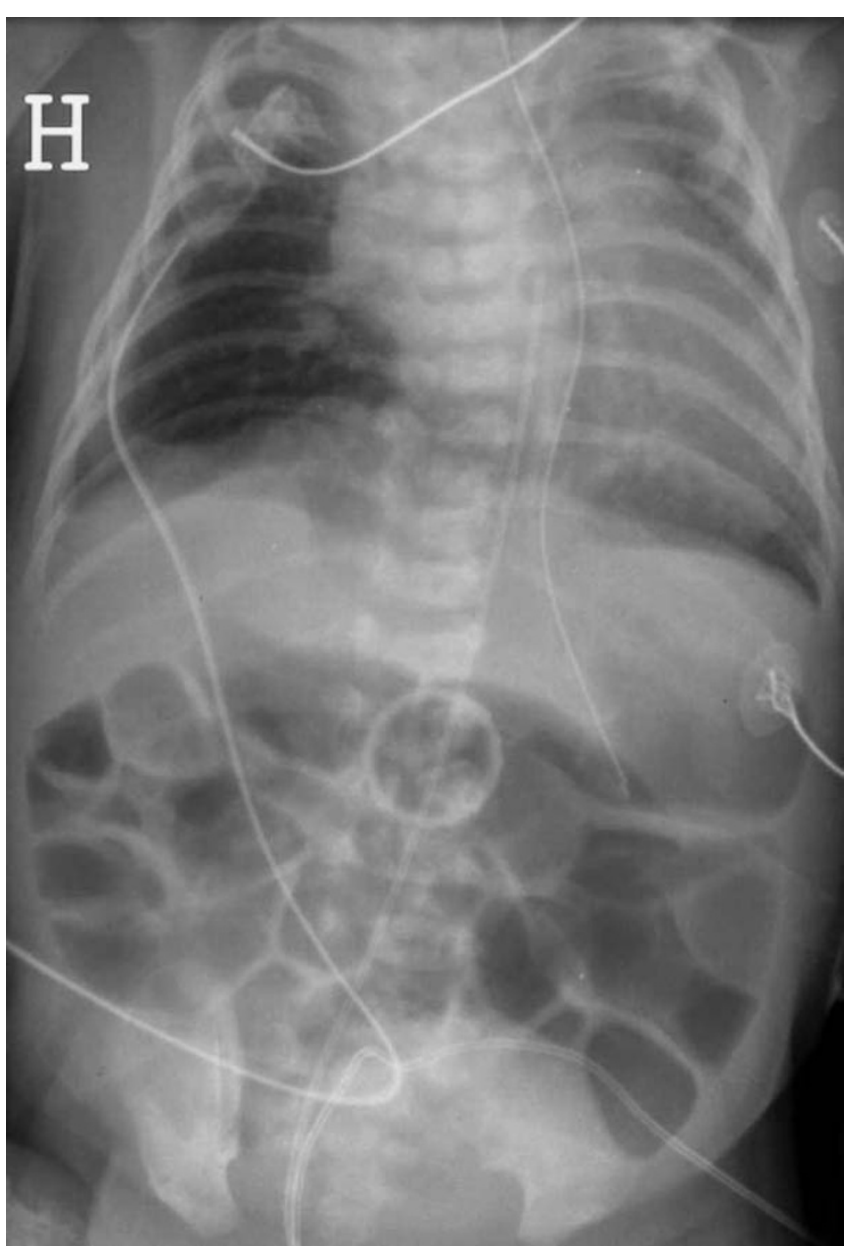

Figure 2

First injection fills a vascular structure draining into the region of the right superior vena cava.

A need for frequent blood sampling and intensive care preoperatively led to the insertion of umbilical arterial and venous catheters. Chest X-ray examination showed an abnormal course of the venous catheter (Figure 1). Perforation of the umbilical catheter into the thoracic cavity was suspected, but blood could be freely drawn. Contrast medium injection under fluoroscopic control demonstrated intravascular position of the tip of the catheter (Figure 2). Two veins joined this vessel, coming in obliquely from the left side of the thorax (Figure 3 ). The vessel drained via the right superior vena cava to the right atrium. The catheter was pulled out under ongoing injection of contrast medium in order to visualise the abnormal course of the vein. There was no connection to the inferior vena cava. The umbilical vein coursed ventrally towards the right-sided lung sinus, and then passed obliquely and medially merging with the venous 


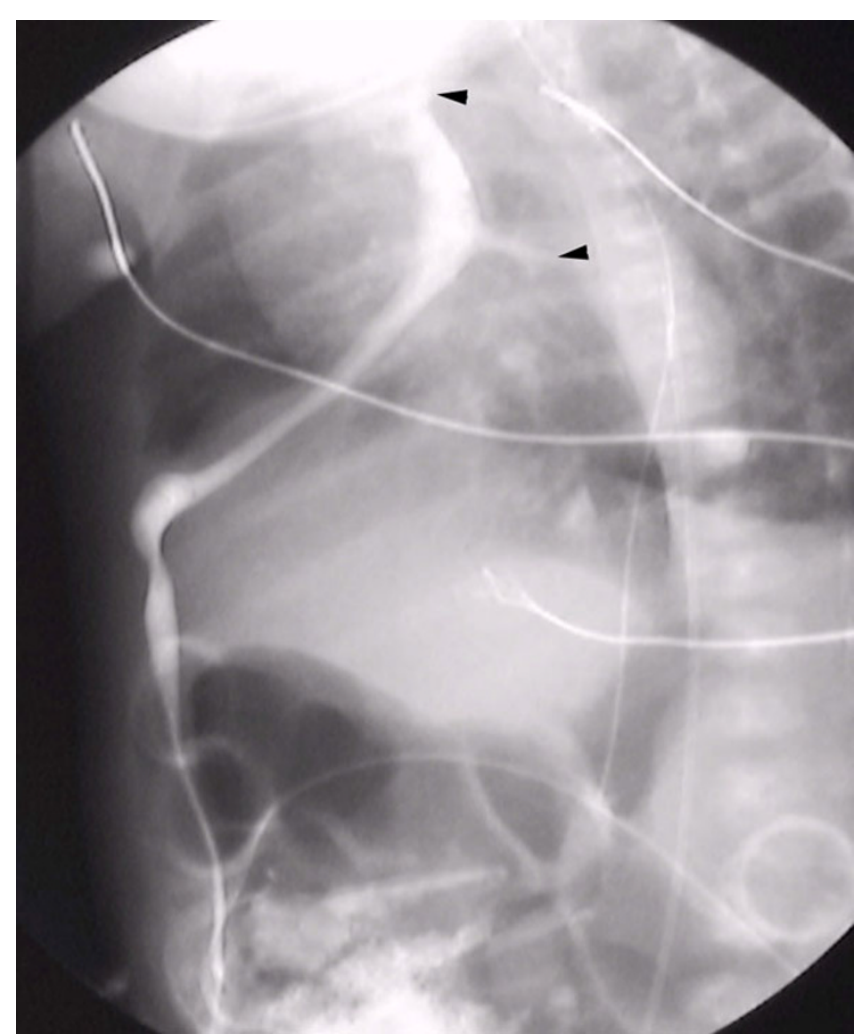

Figure 3

Phlebography shows the abnormal vein coursing from the umbilicus to the right of the liver and emptying into the superior vena cava. Retrograde filling of two smaller veins from the left side (small arrows).

structures described above, before entering the superior vena cava.

The patient had recurrent episodes of pulmonary hypertension, and a corrective operation had to be postponed until the child was seven days old. During surgery, a persistent left-sided superior vena cava was noted. After establishing a graft connection from the right ventricle to the pulmonary arteries the patient was severely unstable and she died three days postoperatively due to cardiac failure.

\section{Conclusions}

The development of the great systemic veins is a complex, but also very interesting process. Few organ systems in the body are so subject to variations and abnormalities in their final, fully developed state. Knowledge of some of the resulting variations may be of clinical importance.

In the very young embryo, the major veins develop from an initially plexiform bed as a number of channels which run mainly in the longitudinal direction. The venous end of the heart receives the vitelline veins from the yolk sac, the umbilical vein from the capillaries of the chorionic villi, and a common cardinal vein from the tissues of the body [6]. From this network the centrally located veins develop. The anterior cardinal veins drain the cephalic end of the embryo where the superior vena cava is formed from the terminal portion of the right anterior cardinal vein and the right common cardinal vein. The latter is formed by fusion of the anterior and the posterior cardinal vein draining the caudal end of the embryo. The subcardinal veins that are located ventrally, form anastomoses with the cardinal veins and may have been involved in the formation of the ventrally located abnormal vein in this patient.

The venous return is preferentially channelled to the right side. Normally, much of the complex network of veins that develops, becomes atretic or is retained as small vessels. The inferior vena cava is formed from the right vitelline vein, and the right umbilical vein becomes atretic. Failure in the junction of the right subcardinal vein with the hepatic vein results in interruption of the inferior vena cava and the systemic drainage will be through the azygos vein to the superior vena cava with the hepatic veins connected directly to the right atrium [6]. In this case, the umbilical vein coursed ventrally indicating a connection between an interrupted inferior vena cava, a persistent right umbilical vein and a persistent cardinal vein.

It has been reported that the absence of the ductus venosus may be associated with different morphological patterns: drainage of a left or a persisting right umbilical vein into an internal iliac vein, the inferior vena cava, or directly into the right atrium [7]. Because the liver is entirely bypassed, the unrestricted umbilical venous blood flow to the heart may result in foetal congestive heart failure. Another pattern of abnormal venous circulation is the umbilical vein connecting to the portal circulation without giving rise to the ductus venosus [8]. Additional cardiac and extracardiac anomalies are reported in foetuses with ductus venosus agenesis [7-9].

An alternative explanation may be that the anastomoses between the two subcardinal veins fails to form normally [10]. The venous return from the lower body will not pass through an intrahepatic portion of the inferior vena cava, but will develop as a persistence of some other embryonic venous pathway, usually as a vena azygos continuation. An azygos continuation may explain why a persistent right umbilical vein drained to the superior vena cava as the cardinal end of the azygos vein and together with parts of the right precardinal vein forms the upper part of the superior vena cava. 
A persistent right umbilical vein is a rare anomaly found in $0,2 \%$ to $0,4 \%$ of foetuses undergoing screening prenatal ultrasonography $[1,2]$. In the embryo the umbilical veins pass laterally to the paired vitelline veins which themselves become connected by anastomoses. They are broken down into intercommunicating sinusoids and may fuse with the right umbilical vein, establishing a venous connection cranial to the interrupted vena cava inferior. In this patient the right vitelline sinusoids may have connected to the right umbilical vein, resulting in a direct communication between the vitelline sinusoids, a persistent right umbilical vein and the network of cardinal veins such as the ventrally located subcardinal and anterior cardinal veins. This may explain the abnormal venous connection demonstrated in this patient.

An accurate description of abnormal venous connections is important when an operation is needed for palliation or correction. Injection of contrast medium in this newborn with a congenital heart defect revealed a very peculiar venous connection that bypassed the liver and passed anterio-laterally through the right hemithorax before crossing in an oblique fashion towards the superior vena cava. We speculate that in the embryo a direct communication has been formed between the vitelline sinusoids, a persistent right umbilical vein and the network of cardinal veins.

\section{List of abbreviations}

UVC umbilical vein catheter, UAC umbilical artery catheter, SVC superior vena cava.

\section{Competing interests}

None declared.

\section{Authors' contributions}

$\mathrm{BN}$ is the neonatalogist that provided care during the admission and composed the manuscript. BS performed and interpreted the radiologic studies.

\section{Acknowledgements}

Text for this section.

\section{References}

I. Lai WW: Prenatal diagnosis of abnormal persistence of the right or left umbilical vein: report of 4 cases and literature review. J Am Soc Echocardiogr 1998, I I:905-9.

2. Wolman I, Gull I, Fait G, Amster R, Kupferminc MJ, Lessing JB, Jaffa AJ: Persistent right umbilical vein: incidence and significance. Ultrasound Obstet Gynecol 2002, 19:562-4.

3. Kinare AS, Ambardekar ST, Bhattacharya D, Pande SA: Prenatal diagnosis with ultrasound anomalous course of the umbilical vein and its relationship to fetal outcome. J Clin Ultrasound 1996, 24:333-8.

4. Kirsch CF, Feldstein VA, Goldstein RB, Filly RA: Persistent intrahepatic right umbilical vein: a prenatal sonographic series without significant anomalies. J Ultrasound Med 1996, I 5:37|-74.

5. De Catte L, Osmanagaoglu K, Schrivjer ID: Persistent Umbilical vein in Trisomy 18: sonographic observation. J Ultrasound Med 1998, I 7:775-779.
6. Arey LB: Developmental anatomy. 7th edition. Philadelphia: WB Saunders; 1965:367-68.

7. Jaeggi ET, Fouron J-C, Hornberger LK, Proulx F, Oberhansli I, Yoo S], Fermont $\mathrm{L}$ : Agenesis of the ductus venosus that is associated with extrahepatic umbilical vein drainage: prenatal features and clinical outcome. Am J Obstet Gynecol 2002, 187: I03I-7.

8. Contratti G, Banzi C, Ghi T, Perolo A, Pilu G, Visentin A: Absence of the ductus venosus: report of 10 new cases and review of the literature. Ultrasound Obstet Gynecol 200I, I 8:605-9.

9. Ricklan DE, Collett TA, Lyness SK: Umbilical vein variations : review of the literature and a case report of a persistent right umbilical vein. Teratology 1988, 37:95-100.

10. Colwin EV: Cardiac embryology. In The science and practice of pediatric cardiology Volume I. Edited by: Garson A, Bricker JT, McNamara DG. Philadelphia/London: Lea \& Febiger; 1990:71-I08.

II. Gray H, Williams PL: Gray's anatomy. Edinburgh, London, Melbourne and New York: Churchill Livingstone 1989:220-6.

\section{Pre-publication history}

The pre-publication history for this paper can be accessed here:

http://www.biomedcentral.com/1471-2431/4/7/prepub

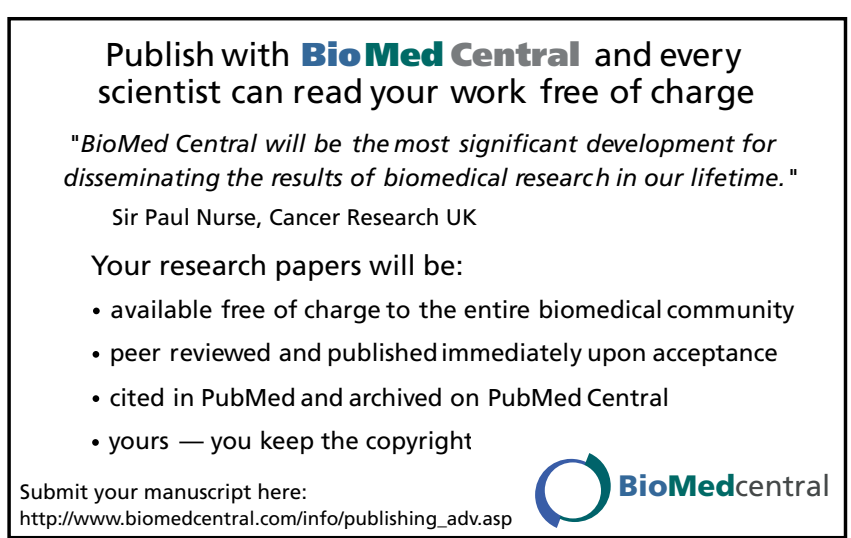

